\title{
Evaluation of the third- and fourth-generation GOCE Earth gravity field models with Australian terrestrial gravity data in spherical harmonics
}

\author{
Moritz Rexer ${ }^{1,2}$ - Christian Hirt ${ }^{1}$ - Roland Pail ${ }^{2}$ - Sten \\ Claessens $^{1}$
}

Received: 09-05-2013 / Accepted: 17-11-2013

\begin{abstract}
In March 2013 the fourth generation of ESA's (European Space Agency) global gravity field models, DIR4 (Bruinsma et al, 2010b) and TIM4 (Pail et al, 2010), generated from the GOCE (Gravity field and steady-state Ocean Circulation Explorer) gravity observation satellite were released. We evaluate the models using an independent ground truth data set of gravity anomalies over Australia. Combined with GRACE ( Gravity Recovery and Climate Experiment) satellite gravity, a new gravity model is obtained that is used to perform comparisons with GOCE models in spherical harmonics. Over Australia, the new gravity model proves to have significantly higher accuracy in the degrees below 120 as compared to EGM2008 and seems to be at least comparable to the accuracy of this model between degree 150 and degree 260. Comparisons in terms of residual quasi-geoid heights, gravity disturbances, and radial gravity gradients evaluated on the ellipsoid and at approximate GOCE
\end{abstract}

\footnotetext{
1 Western Australian Centre for Geodesy, Curtin University of Technology

GPO Box U1987, Perth, WA 6845

Tel.: +61-(0)8-9266-7566

Fax: +61-(0)8-9266-2703

E-mail: m.rexer@tum.de, c.hirt@curtin.edu.au, s.claessens@curtin.edu.au

2 Institute for Astronomical and Physical Geodesy, Technische Universität München

Arcisstrasse 21, D-80333 München

Tel.: +49-(0)89-289-23190

Fax: +49-(0)89-289-23178

E-mail: m.rexer@tum.de, pail@tum.de
}

mean satellite altitude $(\mathrm{h}=250 \mathrm{~km})$ show both fourth generation models to improve significantly w.r.t. their predecessors. Relatively, we find a root-meansquare improvement of $39 \%$ for the DIR4 and $23 \%$ for TIM4 over the respective third release models at a spatial scale of $100 \mathrm{~km}$ (degree 200). In terms of absolute errors TIM4 is found to perform slightly better in the bands from degree 120 up to degree 160 and DIR4 is found to perform slightly better than TIM4 from degree 170 up to degree 250. Our analyses cannot confirm the DIR4 formal error of $1 \mathrm{~cm}$ geoid height $(0.35 \mathrm{mGal}$ in terms of gravity) at degree 200. The formal errors of TIM4, with 3.2 $\mathrm{cm}$ geoid height $(0.9 \mathrm{mGal}$ in terms of gravity) at degree 200, seem to be realistic. Due to combination with GRACE and SLR data, the DIR models, at satellite altitude, clearly show lower RMS values compared to TIM models in the long wavelength part of the spectrum (below degree and order 120). Our study shows different spectral sensitivity of different functionals at ground level and at GOCE satellite altitude and establishes the link among these findings and the Meissl scheme (Rummel and van Gelderen, 1995).

Keywords GOCE · global gravity field model • DIR · TIM · spherical harmonic analysis · coefficient transformation method - Meissl scheme 


\section{Introduction}

Today, a great variety of global gravity field models (GGFMs) generated from data of ESA's (European Space Agency) GOCE (Gravity field and steady-state Ocean Circulation Explorer) gravity field observation satellite (ESA, 1999) exists. Three different approaches for recovering gravity from the satellite's measurements, namely the space-wise (SPW) (Migliaccio et al, 2010), the time-wise (TIM) (Pail et al, 2010), and the direct (DIR) (Bruinsma et al, 2010b) method, have been developed, embedded in the ESA High-level Processing Facility (HPF). In March 2013 the fourth generation models of the DIR and TIM approach were published, both effectively relying on more than 26 months of data. From the SPW approach, however, only two early release models exist, which in the following are not considered further.

Looking at the third and fourth generation models of the DIR and TIM approach, not only the amount of data being used differs with respect to their predecessors, but also the processing strategies applied. Due to those changes improvement may be expected for the new generation models, however, investigations are required. This study evaluates the models' performance in terms of relative improvement and absolute accuracy and shall assess the models' formal error estimates.

GOCE gravity models up to the third generation have been evaluated in many publications with different methods and different datasets. A sound description and comparison of the different processing strategies and the performance of the first generation gravity field models can be found in Pail et al (2011a). In Gruber et al (2011) the first-generation GOCE GGFMs are assessed globally by means of orbit determination of low-orbiting satellites and regionally by point-wise geoid heights from GPS-levelling data. In Hirt et al (2011) first generation GOCE GGFMs are evaluated regionally with terrestrial gravity measurements and point-wise astrogeodetic vertical deflections, and globally with quasi-geoid heights derived from EGM2008. In order to overcome the spectral band limitation of the models the so called spectral enhancement method (SEM) (see, e.g., Hirt et al (2011)) was applied, where information of high frequency GGFMs (like EGM2008 (Pavlis et al, 2012)) and residual terrain data (to account for the ultra-high frequencies) is used to make the spectral content of GGFMs and ground truth data largely compatible. In Tscherning and Arabelos (2011) the first- and second-release GOCE models are compared to gravity anomalies and to radial gradients recovered from GOCE gradiometer data using Least-Squares Collocation (LSC), and to ground truth data sets in various regions of the planet. Janák and Pitoňák (2011) evaluated the first- and second-release GOCE GGFMs with GNSS/levelling data and gravity observations at 31 stations of the Slovak Terrestrial Reference Frame, and additionally compared the models with EGM2008 and GOCO02 (Pail et al, 2011b) in spatial domain making use of a simple version of the SEM. In Hirt et al (2012) gravity signals as implied by the Earth's topography and explained by different isostatic models are used to evaluate the performance of the first- to third-generation GOCE models at various spatial scales. Šprlák et al (2012) evaluated the first- to third-generation models with an independent data set of SEMreduced free-air gravity anomalies in Norway and Bouman and Fuchs (2012) assessed the quality and the performance of the GOCE GGFMs and of the underlying processing strategies with band-filtered gradient observations of the GOCE gradiometer, globally. We also acknowledge other existing studies evaluating GOCE GGFMs over different regions with different terrestrial data sets, e.g. over parts of Sudan (Abdalla et al, 2012), Brazil (Guimarães et al, 2012), Hungary (Szücz, 2012), Norway (Šprlák et al, 2011; Gerlach et al, 2013) and Germany (Voigt et al, 2010; Voigt and Denker, 2011).

The idea and the scope of this study is to evaluate the GOCE gravity field models up to the fourth generation with a new spherical harmonic 
gravity field model, which is independent of GOCE data and contains terrestrial gravity information in Australia. Using a new and independent model of the disturbing potential parameterized in spherical harmonics offers a number of advantages over using just (regional) point or interpolated (gridded) ground truth data sets for an evaluation. First, there is no restriction to a certain gravity field functional, which would normally be predetermined by the type of available ground truth data. As will be shown in this paper, the combined use of different gravity field functionals facilitates a more complete evaluation of the GOCE gravity fields. Different functionals, e.g. gravity disturbances or gravity gradients, have different sensitivity to different spectral bands of the Earth's gravity field and provide valuable complementary information on the GOCE model performance. This has been already noticed, e.g., in Szücz (2012), but the sensitivity of different functionals has not been analysed systematically. Second, the evaluation is not restricted to the exact position of the measurement on ground, but can be freely chosen by a triplet of spherical geocentric coordinates $(\phi, \lambda, r)$ in the spherical harmonic synthesis (SHS). This allows, e.g, straightforward evaluation at ground level and/or at satellite height. Third, comparisons in spherical harmonics avoid the need to overcome a spectral gap, which usually occurs when comparing truncated/band-limited GOCE GGFMs with terrestrial gravity (see SEM approach, e.g. Hirt et al (2011); Šprlák et al (2012)). The SEM, however, is not flexible but restricted to the gravity field functional represented by the comparison data on ground level. Alternatively to the SEM, the terrestrial data (or satellite observations) can be lowpass filtered, e.g., with a Butterworth filter in the frequency domain (Šprlák et al, 2011), a Gaussian filter in the spatial domain (Voigt et al, 2010; Voigt and Denker, 2011), or by means of wavelet approaches like the second generation wavelets approach (Ihde et al, 2010), in order to make them comparable to the band-limited GGFMs.

Having the points outlined above in mind, an elegant way to evaluate a GGFM is by compari- son with another independent GGFM as a reference. Such a data set in principle is already given, e.g., by EGM2008. However, this model does not include all up-to-date gravity data which is, e.g., available for Australia, today.

In section 2 an overview (of the features) of ESA's most recent GOCE gravity field models is provided and the changes between the releases are summarized. In section 3 one way of generating a (comparison) GGFM, which we use to evaluate GOCE's GGFMs above the landmasses of Australia, is presented. A so far little used but effective spherical harmonic analysis (SHA) approach, the so-called coefficient transformation method (Claessens, 2006), is used to retrieve spherical harmonic coefficients of the disturbing potential (see section 3.2). This technique is applied to a global grid of free-air gravity anomalies, which includes terrestrial data over Australia (see section 3.1). The resulting GGFM is then combined with GRACE (Gravity Recovery and Climate Experiment) (Tapley and Reigber, 2001) data on the basis of normal equations (see section 3.3). The finally created set of spherical harmonic coefficients, named AUS-GGM, and its features are discussed in section 3.4. In a next step GOCE GGFMs are evaluated over Australia (see section 4) by means of root-mean-square (RMS) errors (see section 3.5) of residual quasi-geoid heights, gravity disturbances and radial gravity gradients (in spherical approximation). The evaluation is based on three gravity functionals of different spectral sensitivity, evaluated on the ellipsoid (section 4.1) and at satellite height (section 4.2), which allows an interpretation of the results in line with the Meissl-scheme (Rummel and van Gelderen, 1995) in section 4.3. Finally, section 5 summarizes our investigations, and key findings are formulated.

\section{GOCE global gravity field models}

In this section a short overview of the second-, third-, and fourth-generation ESA GOCE models of the DIR and TIM approach is given, focusing on the innovation of each release. A general overview 


\begin{tabular}{|c|c|c|c|c|}
\hline $\begin{array}{l}\text { Model } \\
\text { Name }\end{array}$ & $\mathbf{L}_{\max }$ & Data Used & $\begin{array}{c}\text { A Priori Information / } \\
\text { Constraints }\end{array}$ & $\begin{array}{c}\text { Processing Changes } \\
\text { w.r.t. Previous Release }\end{array}$ \\
\hline DIR2 & 240 & GOCE (8 months) & $\begin{array}{c}\text { ITG-GRACE2010s } \leq \text { d/o } 150 \\
\text { spherical cap reg. } \\
\text { (using GRACE/LAGEOS) }\end{array}$ & $\begin{array}{l}\text { SGG: } 10-125 \mathrm{mHz} \text { bandpass filter } \\
\text { SST: } \leq \mathrm{d} / \mathrm{o} 130\end{array}$ \\
\hline DIR3 & 240 & $\begin{array}{l}\text { GOCE (18 months) } \\
\text { GRACE (6.5 years) } \\
\text { SLR: LAGEOS- } 1 /-2 \\
\text { ( } 6.5 \text { years })\end{array}$ & $\begin{array}{c}\text { Kaula } \geq \text { degree } 200 \\
\text { DIR } 2 \leq \mathrm{d} / \text { o } 240 \\
\text { spherical cap reg. } \\
\text { (using GRACE/LAGEOS) } \\
\end{array}$ & $\begin{array}{c}\text { GRACE as normal eq. } \\
\text { LAGEOS as normal eq. } \\
\text { SGG components : } \\
\text { equal relative weights }\end{array}$ \\
\hline DIR4 & 260 & $\begin{array}{l}\text { GOCE (33 months) } \\
\text { GRACE (9 years ) } \\
\text { SLR: LAGEOS- } 1 /-2 \\
\quad(1985-2010)\end{array}$ & $\begin{array}{c}\text { Kaula } \geq \text { degree } 200 \\
\text { DIR } 3 \leq \mathrm{d} / \text { o } 240 \\
\text { spherical cap reg. } \\
\text { (using GRACE/LAGEOS) }\end{array}$ & $\begin{array}{l}\text { SGG: inclusion of } V_{x z} \text {-component } \\
\text { SGG: } 8.3-125 \mathrm{mHz} \text { bandpass filter } \\
\text { GRACE: GRGS-RL02 up to d/o } 54 \\
\text { GRACE: GFZ-RL05 from degree } 55\end{array}$ \\
\hline TIM2 & 250 & GOCE (8 months) & $\begin{array}{l}\text { Kaula } \geq \text { degree } 180 \\
\text { spherical cap reg. } \\
\text { (using Kaula's rule) }\end{array}$ & none \\
\hline TIM3 & 250 & GOCE (18 months) & $\begin{array}{l}\text { Kaula } \geq \text { degree } 180 \\
\text { spherical cap reg. } \\
\text { (using Kaula's rule) }\end{array}$ & SGG: inclusion of $V_{x z}$-component \\
\hline TIM4 & 250 & GOCE (32 months) & $\begin{array}{l}\text { Kaula } \geq \text { degree } 180 \\
\text { spherical cap reg. } \\
\text { (using Kaula's rule) }\end{array}$ & SST : short-arc integral \\
\hline
\end{tabular}

Table 1 Main characteristics of a selection of the most recent gravity field models relying on GOCE data (periods do not reflect the effective amount of data being used) [source: ICGEM (http://icgem.gfz-potsdam.de/ICGEM/ICGEM.html)]; $L_{\max }$ denotes the maximum spherical harmonic degree of the model; SGG : satellite gravity gradiometer; SST : satellite-to-satellite tracking; SLR : satellite laser-ranging; DIR: GO_CONS_GCF_2_DIR_R $(2,3,4)$; TIM: GO_CONS_GCF_2_TIM_R $(2,3,4)$

on the underlying principles and methods of the two approaches can be found, e.g., in Pail et al (2010, 2011a); Bruinsma et al (2010b). Table 1 lists the main characteristics of the models and changes with respect to their previous releases (right column). The information was retrieved from the models' header information and their respective data sheets, all released via the ICGEM-homepage (http://icgem.gfz-potsdam.de/ICGEM/).

DIR models of second and third generation have a maximum spherical harmonic degree $L_{\max }$ of 240, while the DIR4 model has a higher spatial resolution $\left(L_{\max }=260\right)$. All three DIR releases (in addition to GOCE gravity gradient data) contain GRACE information in the lower to medium range spherical harmonic degrees. In the second DIR release the ITG-GRACE2010s (Mayer-Gürr et al, 2010) solution is introduced as a priori information until degree and order (d/o) 150. In the DIR3 and DIR4 models GRACE is combined with GOCE and satellite laser-ranging (SLR) data of LAGEOS (Tapley et al, 1993) on the basis of normal equations. In the DIR3 model GRACE normal equations up to d/o 160 are used which entirely rely on the procedures of the second release CNES/GRGS (Centre National d'Etudes
Spatiales/Groupe de Recherches de Géodésie Spatiale) models (Bruinsma et al, 2010a). In the DIR4 model, the same GRGS-GRACE normal equations are used only up to d/o 54. From degree 55 up to degree 180 DIR4 is based on GRACE GFZ (GeoForschungsZentrum Potsdam) release 5 gravity field solution (Dahle et al, 2013). The amount of data/observations from all involved satellites is increasing with each DIR release. Effectively, DIR3 and DIR4 is based on 12 months and 27.9 months of GOCE data, respectively. In the last three DIR releases consistently a spherical cap regularization (SCR) (Metzler and Pail, 2005) was applied using GRACE and LAGEOS information in order to overcome GOCE's polar observation gap (Sneeuw and van Gelderen, 1997), which is caused by the satellite's orbit inclination of $96.7^{\circ}$ (ESA, 1999). In the third and fourth DIR release, additionally, the predecessor release was used as a priori information (up to d/o 240) and a Kaula regularization (see, e.g., Metzler and Pail (2005)) was applied starting at degree 200. Since the third DIR release the information gathered from each of the three gravity tensor elements measured with GOCE's on-board SGG is weighted equally in the combination. In the DIR4 release 
information of the off-diagonal tensor element $V_{x z}$ was likewise included. Besides, in DIR4, the spectral band of the bandpass filter used to filter the SGG observations was extended by $1.7 \mathrm{mHz}$ towards the lower frequency domain. Within the DIR approach (in contrary to the TIM approach) the use of GOCE gradient information is restricted to a certain spectral band, which is close to the gradiometer's designed measurement bandwidth (5 mHz to $100 \mathrm{mHz}$, see, e.g. ESA (1999)).

Looking at the TIM models, the models' maximum spherical harmonic degree is constant at degree 250 for the latest three releases. All TIM models exclusively rely on GOCE SGG and SSThl (satellite-to-satellite observation in high-low mode) data, however, the amount of data increases with each release. Effectively, TIM3 and TIM4 are based on 12 months and 26.5 months of GOCE data, respectively. Each TIM model is constrained according to Kaula's rule (Kaula, 1966) by means of (1) a spherical cap regularization (Metzler and Pail, 2005) in order to deal with the polar observation gap (ESA, 1999) and (2) a (full) Kaula regularization starting at degree 180 . Since the first TIM release the stochastic models for the gradient observations are estimated from small, coherent data-patches, resulting in improved (tuned) filtering of the gradients in the time domain. Remaining unchanged for the all releases, the filtering procedure within the TIM approach allows to use the information of the gradient observations over the entire spectrum. Since the TIM3 release, the off-diagonal tensor element $V_{x z}$ finds application in the models. Finally, in the fourth TIM release the processing strategy for the SST normal equations was changed from the energy integral approach (Badura, 2006) to the short-arc integral method (Mayer-Gürr et al, 2006).

Not explicitly included in the table is the introduction of a new Level-1b (L1b) processing procedure (Stummer et al, 2011, 2012) in 2012 due to which a better performance of GOCE's satellite gravity gradiometer (SGG) is to be expected in the fourth generation models (DIR and TIM). According to Pail et al (2012) gradiometry-only gravity field estimates show largest improvements in the recovery of lower and medium degree coefficients and the accuracy of combined gravity field models is reported to gain more than $10 \%$, even in higher degrees, due to the new L1b processing.

\section{Data and methods for the creation of a GOCE-independent comparison GGFM}

\subsection{Data}

The aim of the research is to create a set of global spherical harmonic coefficients of the disturbing potential from gridded (terrestrial) gravity data which is (a) completely independent of GOCE, and (b) of sufficient spatial resolution and accuracy ( $\leq$ 1-2 $\mathrm{cm}$ geoid height or $\leq 1 \mathrm{mGal}$ at a spatial scale of $100 \mathrm{~km}$ ) (c.f. ESA (1999)) in order to evaluate the performance of GOCE GGFM. Globally this cannot be achieved, as there is no observation technique with global coverage and similar or higher performance than GOCE. Regionally, however, it is possible to use terrestrial gravity observations to evaluate GOCE. For our research, a comparison GGFM was computed with terrestrial gravity over the land area of Australia.

The Australian terrestrial gravity data set available for this work consists of gridded Faye free-air gravity anomalies on the topography with a resolution of 1'x1' (arc-minutes). In total about 1.4 million gravity observations over Australia were taken from Australia's National Gravity Database (hosted at Geoscience Australia) to create the gridded data set (Featherstone et al, 2010). This, e.g., exceeds the amount of observations $(905,483)$ which have been used to compute EGM2008 (c.f. Claessens et al (2009)). The 1'x1' anomaly grid has been computed from the database in the course of the country's national (quasi-) geoid AUSGeoidog (Featherstone et al, 2010) computation. The computation and the gridding of the gravity anomalies refers to the procedure originally described in detail in Featherstone and Kirby (2000). Within 


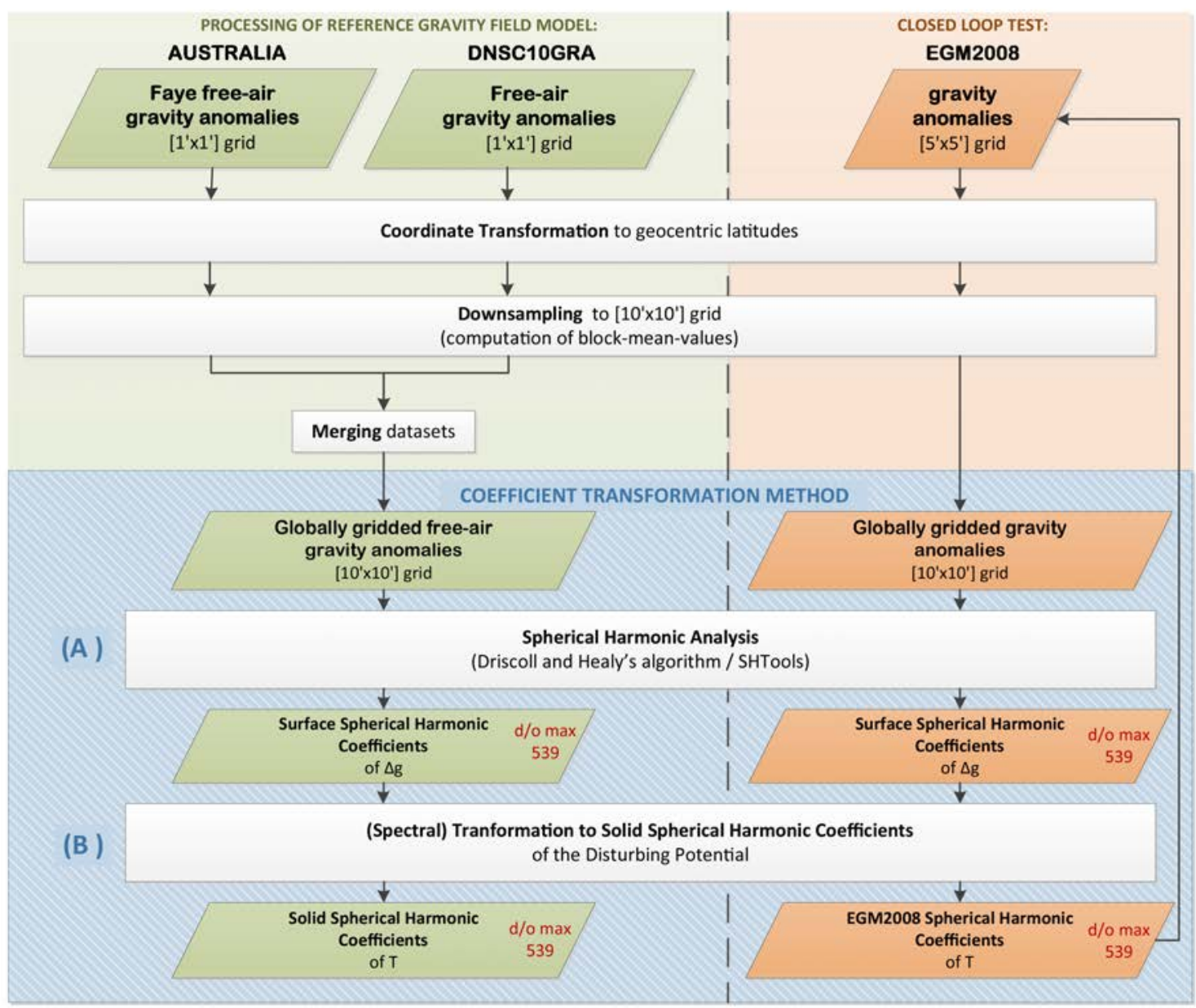

Fig. 1 Processing scheme for the generation of a comparison GGFM (left / green) and scheme for the closed loop test relying on EGM2008 (right / orange)

the approach aliasing errors are minimized by interpolating the observed gravity anomalies after a point-wise subtraction of a simple Bouger anomaly (which is then restored after the interpolation to a grid). The finally obtained Faye free-air anomalies are free-air anomalies of Molodensky's type with the terrain correction applied. The additional terrain correction approximates Molodenski G1 correction term (see, e.g, Torge (2001), p.290; Wang (1989)), which is generally needed for the downward continuation of free-air anomalies to the ellipsoid.

The remainder of the Earth's gravity field is represented by a global grid of gravity anomalies provided by the Technical University of Denmark's
(DTU) marine gravity model DNSC10GRA, which is the successor of DNSC08GRA described in Andersen et al (2009). The DTU data set relies on EGM2008 (Pavlis et al, 2012) over land and ArcGP (Forsberg and Kenyon, 2004) gravity data and ICEsat's laser altimetry data (Zwally et al, 2002) over polar regions. Offshore gravity is recovered from the knowledge of the oceans' mean sea surface height ( $\mathrm{SSH}$ ) derived from satellite altimetry. The mean SSH is determined with a so called double retracking technique (Andersen et al, 2009), which leads to an augmented spatial coverage (especially in ice-covered regions), using data of the altimetry satellites GEOSAT and ERS-1. Data of the altimeter missions Topex/Poseidon, 
GFO, ERS-2 and Envisat also found application in the DNSC10GRA development.

\subsection{Gridding and spherical harmonic analysis}

In this section, the computation steps in order to obtain coefficients of the disturbing potential from the initial data sets are explained. Figure 1 schematically shows the data flow of the processing (left side of the scheme). In a pre-processing step, the data sets have to be consistently prepared and merged for the subsequent SHA procedure by a coordinate transformation and consecutive down-sampling. The SHA is accomplished based on the coefficient transformation method (CTM) (Claessens, 2006). This approach requires (A) a spherical harmonic analysis to compute a set of surface spherical harmonic coefficients and (B) a spectral transformation to transform these into solid spherical harmonic coefficients of the disturbing potential (c.f. Figure 1).

For the spherical harmonic analysis (A), a homogeneous global grid of gravity anomalies on the ellipsoid with an equiangular spacing in both 2D directions is needed. As mentioned above, such a grid with an equiangular spacing of 1 arcminute is given with the DNSC10GRA data set, globally. Over the landmass of Australia, the country's terrestrial gravity anomalies are used while DNSC10GRA is used to describe the Earth's gravity outside of Australia. Before merging the 2 data sets, however, it is necessary to adapt and harmonize the data sets, taking the following considerations into account: The analysis procedure (A) relies on a quadrature algorithm based on Fourier transforms and a sampling theorem, both described by Driscoll and Healy (1994). As defined by the sampling theorem, the maximum spherical harmonic degree $L_{\max }$, that can (exactly) be retrieved from a band-limited function given on a sphere, is defined through

$$
L_{\max }=\frac{N}{2}-1,
$$

where $N$ denotes the even number of point values in latitude direction of an equiangular grid of size $N \mathrm{x} N$ or $N \mathrm{x} 2 N$ (points in latitude direction x points in longitude direction). Here, the latter grid sampling finds application for reasons of convenience, as it is identical to the sampling of the used terrestrial gravity anomaly grid. For the purpose of this study the maximum degree has to be at least equivalent to the GOCE GGFM with the highest resolution, which is given with the fourth generation model of the DIR approach $\left(L_{\max }=260\right)$. Aimed at a maximum spherical harmonic degree of 539 of the final GGFM - which is more than good enough for the purpose of this study - the gravity anomaly grids are down-sampled accordingly to a 10' (arcminutes) spacing (leading to a global grid of 1080x2160 points). The down-sampling is performed by computing block-mean values for all grid-points entirely contained in adjacent, equiangular blocks of 10'x10' size. Prior to the downsampling, the grids have to be transformed from geodetic to geocentric latitudes. This can, e.g., be done with a $2 \mathrm{D}$-spline interpolation using the simple relation

$\tan \Theta=\frac{a^{2}}{b^{2}} \tan \phi$

between the spherical co-latitude $\Theta$ and the geodetic co-latitude $\phi$, where $a$ is the semi-major and $\mathrm{b}$ the semi-minor axis of the underlying ellipsoid (see, e.g., Torge (2001), p.95), which is GRS80 (Moritz, $2000)$ in this case.

The set of spherical harmonic coefficients (SHC), which is computed with the Driscoll and Healy's (DH) algorithm using the SHTOOLS ${ }^{1}$ software, is a set of surface SHCs. It can only be used to retrieve exactly the same gravity functional which was used as input (in this case gravity anomalies). Thus, a subsequent transformation (B) is needed to retrieve solid SHCs of the disturbing potential. This spectral transformation completes the CTM approach, which has been proposed by Claessens (2006). The CTM is used in conjunction with numerical quadrature methods like SHTools' $\mathrm{DH}$-algorithm, and is based on the possibility to describe function values on the ellipsoidal surface in terms of a set of surface SHCs. Further, the CTM

\footnotetext{
1 http://www.ipgp.fr/ zor/SHTOOLS/SHTOOLS.html
} 
proves to be superior to several existing methods and comparable to the ellipsoidal harmonics method (EHM) (Jekeli, 1988) (c.f. Claessens (2006)). To be more precise, the CTM shows better accuracy regarding near-zonal coefficients and is slightly worse regarding the near-sectoral coefficients compared to the EHM. It is shown that the CTM's mean error is $0.3 \mathrm{~mm}$ and its maximum error $2.6 \mathrm{~mm}$ expressed in geoid height (in the spectral range of degree 20 to degree 340) (c.f. Claessens (2006)). For detailed information on the CTM and the transformation we refer to the cited literature, where the algorithm and its performance is comprehensively described.

The function described by the gravity anomaly grid points on the sphere is not band-limited as it is needed for DH's algorithm, and thus aliasing is to be expected. However, this effect can be ignored for the purpose of our research. Closed loop tests with a gravity anomaly grid expanded (up to degree 2190) from the EGM2008 gravity field model, passed through the same procedure outlined above (illustrated on the right side of figure 1), indicate that the input SHCs can be restored with sufficient accuracy. The gravity residuals reach at maximum $\pm 0.75 \mathrm{mGal}$ at degree 200 and their mean between degree 20 to degree 340 is $0.0025 \mathrm{mGal}$. Globally the root-mean-square (RMS) of closed loop discrepancies is $0.068 \mathrm{mGal}$ at a spatial scale of 100 $\mathrm{km}$ (degree 200) and $0.07 \mathrm{mGal}$ at a spatial scale of $80 \mathrm{~km}$ (degree 250) in terms of gravity anomalies. By comparison, the estimated error of GOCE models is about $0.9 \mathrm{mGal}(\mathrm{HPF}, 2013 \mathrm{~b})$ and 0.35 mGal (own computation) at degree 200 for TIM4 and DIR4, respectively.

\subsection{Combination with GRACE}

As a final step in order to obtain SHCs eligible to evaluate GOCE GGFMs, we combine the above received solid SHCs from the CTM with data of the GRACE satellite mission. GRACE information can be seen complementary to the high-frequency terrestrial data (present in Australia), as GRACE shows a very high performance in the recovery of the long wavelength part of the spectrum of the Earth's gravity field. The combination is performed on the basis of full GRACE normal equations (complete up to d/o 180), which have been computed in the course of the ITG-GRACE2010 gravity field model (Mayer-Gürr et al, 2010). The formal error-per-degree estimate of ITG-GRACE2010 at degree 120 is $1.5 \mathrm{~mm}$ (and $4.2 \mathrm{~mm}$ accumulated error from own computations) in terms of geoid heights (c.f. Mayer-Gürr et al (2010)).

The combination can be expressed as a leastsquares problem by introducing a $(i)$ GRACE type system

$l+v_{1}=A \cdot x$

where $l$ are the GRACE observations used in the production of ITG-GRACE2010, $A$ is the design matrix, $x$ is the unknown parameter vector and $v_{1}$ denotes the residuals of the process. Further, we introduce a $(i i)$ system relying entirely on a priori information

$x_{0}+v_{2}=I \cdot x$

where $x_{0}$ a priori known parameter vector, $I$ the identity matrix and $v_{2}$ denotes the residuals of the process. Because of the linearized form and the affiliation to the same set of parameters, system $i$ (equation 3) and system ii (equation 4) can be combined, assuming uncorrelated (pseudo-) observation groups by

$$
\begin{aligned}
& \left(A^{T} \Sigma(l)^{-1} A+I^{T} \Sigma\left(x_{0}\right)^{-1} I\right) \cdot x \\
& =A^{T} \Sigma(l)^{-1} l+I^{T} \Sigma\left(x_{0}\right)^{-1} \cdot x_{0}
\end{aligned}
$$

where $A^{T} \Sigma(l)^{-1} A$ is the ITG-GRACE2010 normal equation matrix, $A^{T} \Sigma(l)^{-1} l$ is the corresponding right-hand side, $\Sigma(l)$ and $\Sigma\left(x_{0}\right)$ denote the variance-covariance matrices of system $i$ and system $i i$, respectively. In our case the a priori known parameters $x_{0}$ are the SHCs related to the terrestrial data grid, and computed by the CTM approach. The variance-covariance matrix $\Sigma\left(x_{0}\right)$ only consists of diagonal elements, the variances of the SHCs. The variances were defined empirically and degree-wise (based on the assumption that 

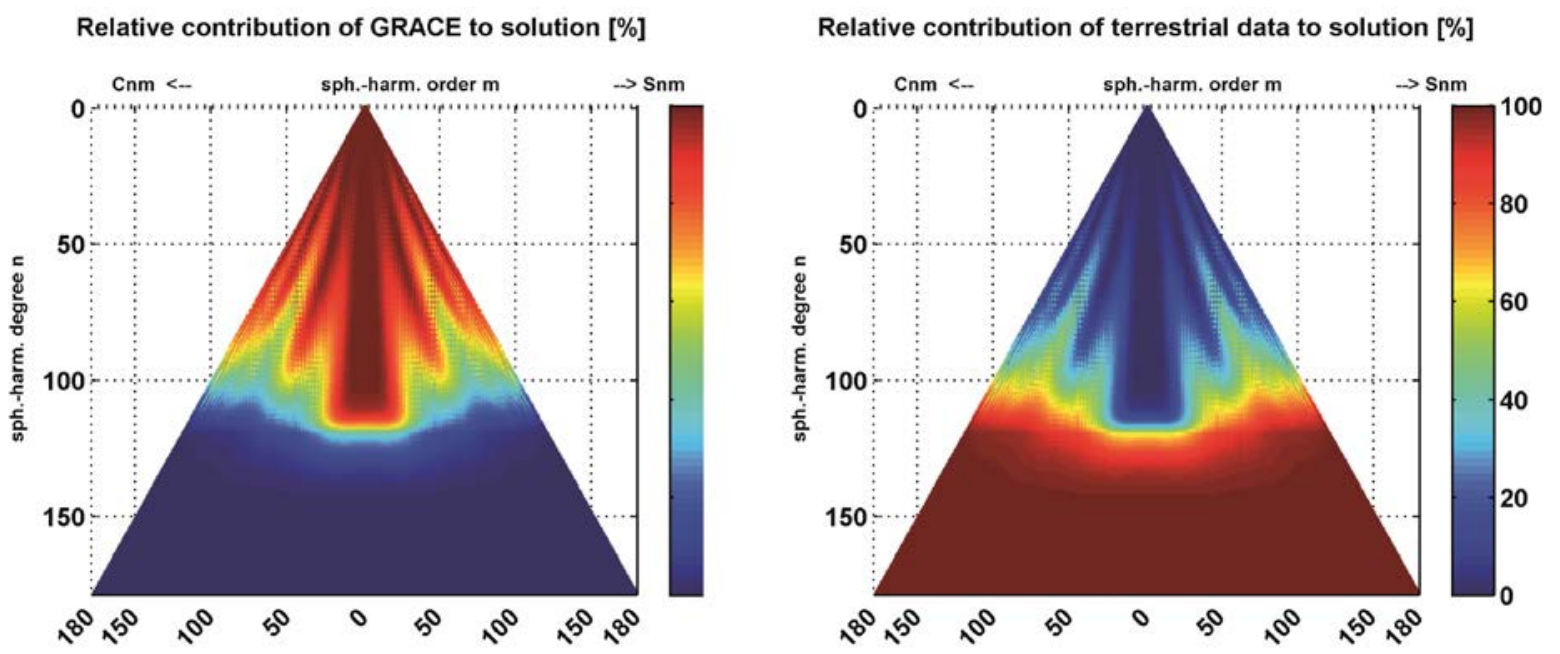

Fig. 2 Contribution of GRACE (left plot) and terrestrial data (right plot) to the combined solution

GRACE provides more accurate information on the long wavelength part of the spectrum), so that their impact in the combination is minor below spherical harmonic degree 120 and dominates beyond degree 120 regarding the given mean GRACE variance (-covariance) information per degree. Expressed numerically in terms of standard deviations $\left(\sigma\left(x_{0}\right)\right)$, we start with $1 \cdot 10^{-10}$ at degree 0 and decrease with an increment of $7.92 \cdot 10^{-13}$ for each degree, reaching $4.149 \cdot 10^{-12}$ at degree 120 (and staying constant up to degree 180). Figure 2 shows the exact ratio of contribution of GRACE information (left side) and terrestrial (and DNSC10GRA) information (right side) per spherical harmonic coefficient. From Figure 2 it becomes clear that the combination consists of terrestrial data (and DNSC10GRA data outside of Australia), solely, beyond the spherical harmonic degree 140.

We have exchanged the zonal spherical harmonic coefficient of degree two (C20) with its equivalent from EGM2008, because GRACE's J2 coefficient is subject to tidal aliasing (c.f., e.g., Chen and Wilson (2010); Lavallée et al (2010)). Within EGM2008 J2 originates from SLR, mainly.

As an aside, discrepancies between the terrestrial Faye free-air gravity anomalies and the DNSC10GRA / EGM2008 free air gravity anomalies over the landmass of Australia could be de- tected, predominantly of long-wavelength character (up to d/o 50). The highest amplitudes can be found along the Great Dividing Range, the mountain chain in Australia's South-East, with 15 mGal. In terms of RMS difference, the discrepancy accounts for $1.6 \mathrm{mGal}$ over Australia. These differences have already been reported to the data producers and warrant further investigations which are considered future work. This observation corroborates our strategy to exclusively use GRACE on the long spatial scales. As to be expected the combined solution then shows better agreement with to EGM2008 below d/o 50 .

\subsection{Features and errors of the comparison GGFM}

In this section the features of the created comparison model AUS-GGM are described in order to judge its ability to evaluate GOCE GGFMs over Australia. The description is based on (approximate) cumulative errors, which formally reflect the models' performance at a specific spherical harmonic degree on a global scale (and not only over Australia). Figure 3 shows the respective cumulative formal geoid error of AUS-GGM (in yellow) together with the equivalent errors of the other GGFMs which find attention in this research. In figure 3, the errors of AUS-GGM's terrestrial gravity (which is incorporated in the model approximately above degree 120 (see section 3.3) and 
which is mainly from DNSC10GRA/ EGM2008) are approximated by the standard deviations which are denoted for EGM2008, as we do not obtain a formal error for the terrestrial gravity data from the CTM. As to be expected from the combination of the terrestrial gravity with ITG-GRACE2010 (see section 3.3), we see the cumulative error of AUS-GGM rise around degree 120 where the terrestrial gravity information supersedes GRACE's information. From degree 2 up to degree 100 the cumulative geoid error of AUS-GGM is smaller or at least comparable to that of DIR4 (blue) and ITG-GRACE2010 (green) and smaller compared to the other illustrated geopotential models. At degree 200 our computations show that AUS-GGM with $35 \mathrm{~mm}$ cumulative geoid error seems comparable to the quality of TIM4 (40 mm) and DIR3 (32 $\mathrm{mm})$. It clearly outperforms TIM3 $(60 \mathrm{~mm})$ and EGM2008 $(72 \mathrm{~mm})$, however, AUS-GGM shows a significantly higher error than DIR4 $(12 \mathrm{~mm})$. In the spectral range from degree 120 up to degree 250 DIR4 is the only model which constantly performs significantly better than AUS-GGM from formal perspective. Bear in mind, however, that the cumulative errors reflect the global error and that the formal error of AUS-GGM is approximate. For Australia, where we inserted dense and accurate terrestrial gravity information, the cumulative errors as displayed in figure 3 are likely to be too pessimistic. From this perspective we conclude that AUS-GGM is well designed to serve as a comparison GGFM over Australia in order to evaluate differences between the GOCE GGFMs and may also be used to give absolute error estimates under consideration of its characteristic cumulative error.

\subsection{Evaluation method}

For the evaluation of GOCE GGFMs with the newly created AUS-GGM in spatial domain, we make use of the harmonic_synth software (Holmes and Pavlis, 2008) to expand the coefficients to grids. Evaluations are performed in the spatial domain and not in frequency domain, as we only want to focus on the landmass of Australia, where

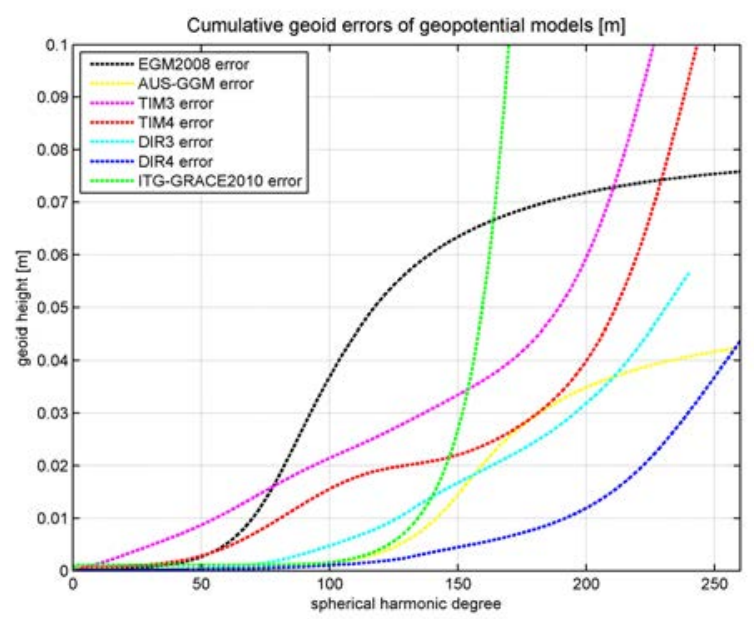

Fig. 3 Cumulative formal error of AUS-GGM (approximate) and other geopotential models in meters of geoid height per spherical harmonic degree

newer terrestrial information has been introduced. A grid-spacing of 10 arcminutes is chosen in the SHS in order to yield an oversampling compared to the maximum degree of GOCE GGFMs $(\leq$ degree 260). Further, all grid values are computed as point values in geodetic coordinates w.r.t. the GRS80 (Moritz, 2000) ellipsoid.

All RMS values are computed from the differences of the AUS-GGM grid and the GOCE GGFM grid under evaluation, w.r.t. the underlying gravity functional. All grid-points outside of Australia's landmass are not considered in the RMS. The RMS is cumulative in the sense that the spherical harmonic expansion in the synthesis was always done starting at degree 2 up to the denoted maximum spherical harmonic degree.

\section{Results and Discussion}

As outlined in the introduction, we focus on the evaluation of the third- and fourth-generation GOCE GGFMs. In section 4.1 the evaluation is done on the ellipsoid, in section 4.2 at an approximate GOCE altitude of $h=250 \mathrm{~km}$.

The gravity functionals under evaluation are the quasi-geoid height $\zeta$ in meter $[\mathrm{m}]$, the gravity disturbance $T_{r}$ (first radial derivative of the disturbing potential) in milli-Gal [mGal] $(1 \mathrm{mGal}=$ $10^{-5} \frac{\mathrm{m}}{\mathrm{s}^{2}}$ ), and the radial gravity gradient $T_{r r}$ (sec- 


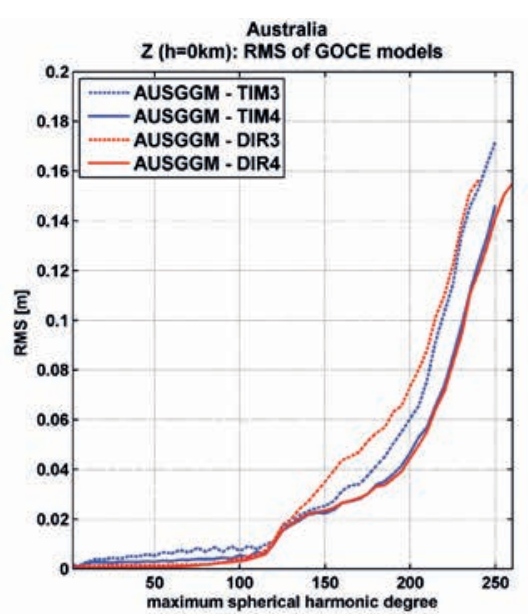

a) quasi-geoid heights

Australia

$\mathbf{Z}(\mathrm{h}=0 \mathrm{~km})$ : RMS of GOCE models (zoom)

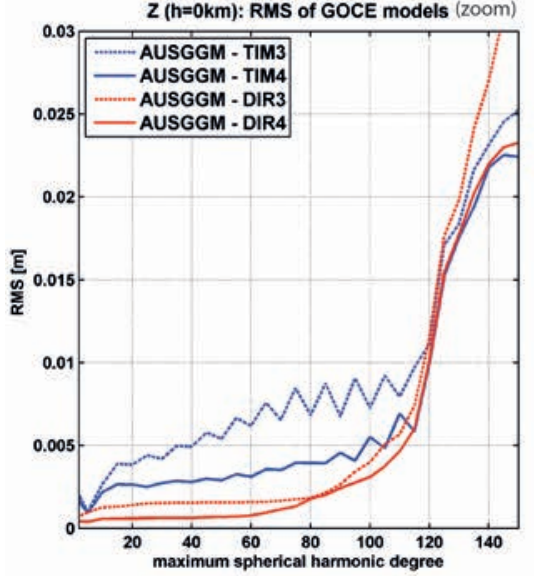

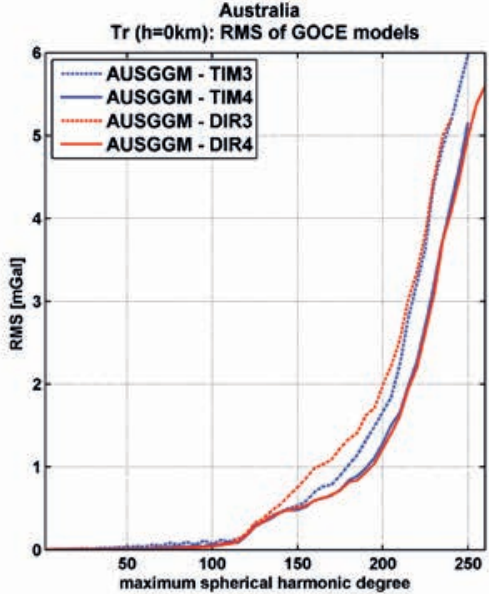

b) gravity disturbances

Australia
$\operatorname{Tr}(\mathrm{h}=0 \mathrm{~km})$ : RMS of GOCE models (zoom

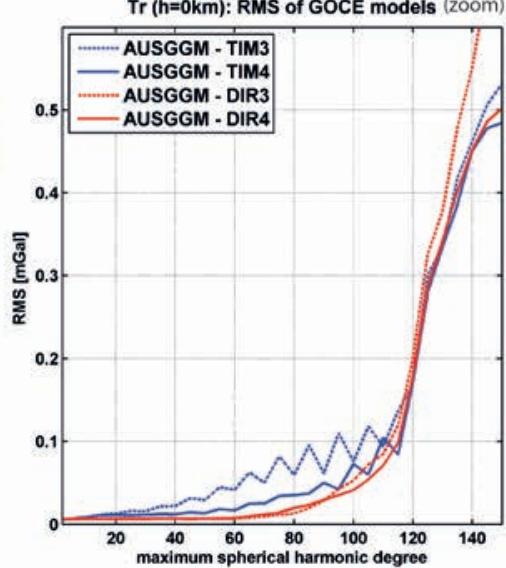

Australia

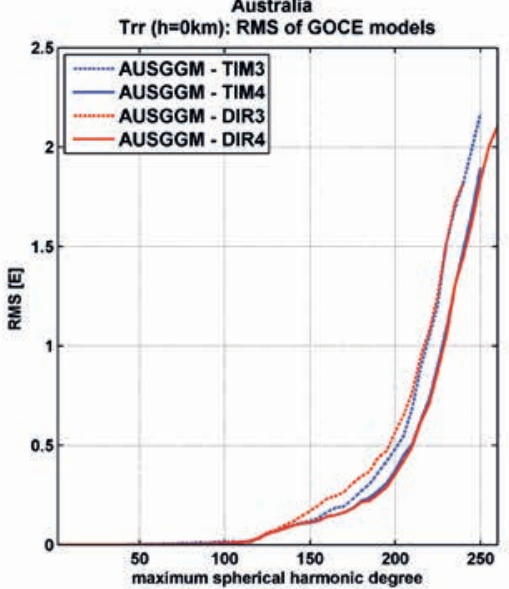

C) radial gravity gradient

Australia

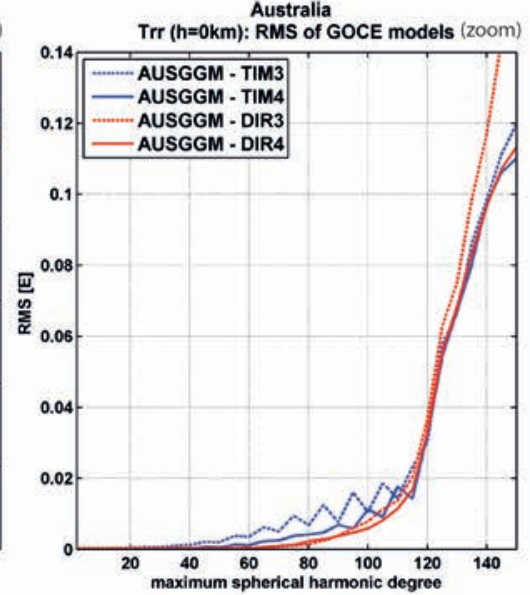

Fig. 4 RMS values computed from the differences of selected GOCE GGFMs and the newly retrieved AUS-GGM in terms of (a) quasi-geoid heights $\zeta$ in meters [left], (b) gravity disturbances $T_{r}$ in mGal [middle] and (c) radial gravity gradients $T_{r r}$ [right] on the ellipsoid $(h=0)$; the bottom row plots zoom into the respective upper plot in the degree range 0 to 150

ond radial derivative of the disturbing potential) in Eötvös [E] $\left(1 E=10^{-9} \frac{1}{s^{2}}\right)$, all in spherical approximation. With this set-up we intend to follow the Meissl scheme (Rummel and van Gelderen, 1995) and investigate the models' performances in each of the six domains of the Meissl scheme.

\subsection{Evaluation on ground level}

With the evaluation on ground level (= surface of GRS80 ellipsoid) we intend to verify the accuracy of the models at a height which is representative for applications of GOCE data on land (e.g. levelling).

In figure 4 the RMS values over Australia of the GOCE GGFMs w.r.t. AUS-GGM are displayed for all three gravity functionals expanded to different maximum degrees. Analyzing all three plots in figure 4, one can clearly see that the fourth generation models TIM4 and DIR4 outperform their respective predecessors beyond degree 150 . In the spectral range starting at degree 120 up to degree 250 both models show very similar RMS behavior. TIM4 seems to perform marginally better between degree 120 and degree 160 ( $\leq 4 \%$ RMS difference) and DIR4 seems to perform marginally better ( $\leq 6 \%$ RMS difference) in the bands from degree 170 to degree 250 . The latter might be explained by the fact that DIR4 holds one additional month of GOCE information compared to TIM4 (see table 1 in section 2). Table 2 gives the RMS values for each model at the spatial scale of 100 $\mathrm{km}$ half wavelength (= degree 200) for the three functionals. Given those values TIM4 shows an 
average relative improvement of about $23 \%$ w.r.t. TIM3 and DIR4 shows an average relative improvement of about $39 \%$ w.r.t. DIR3.

Compared absolutely in terms of geoid heights $\zeta$, the calculated RMS for DIR4 at degree 200 $(4.5 \mathrm{~cm})$ is slightly lower than that of TIM4 $(4.7 \mathrm{~cm})$. The absolute (formal) error at degree 200 is officially denoted $1 \mathrm{~cm}$ in geoid height for DIR4 (HPF, 2013a) and $3.2 \mathrm{~cm}$ in geoid height for TIM4 (HPF, 2013b) (our own computations show a cumulated geoid error of $1.2 \mathrm{~cm}$ and 4 $\mathrm{cm}$ for DIR4 and TIM4, respectively). Thus, our calculated RMS values at degree 200 exceed both models' formal errors by $3.5 \mathrm{~cm}$ and 1.5 cm for DIR4 and TIM4, respectively. However, the RMS values from the differences reflect the errors of both involved data sets, the $(i)$ GOCE models and $(i i)$ the GRACE / terrestrial data in the AUS-GGM model. Having this in mind and considering that the observed TIM4 RMS is very close to the RMS error of $4.5 \mathrm{~cm}$, which has been estimated for TIM4 independently from comparisons to 675 GPS/levelling observations in Germany at degree 200 (HPF, 2013b) our retrieved RMS for TIM4 over Australia seems to be plausible and the TIM4 formal error estimate of $3.2 \mathrm{~cm}$ seems to be quite realistic. In the case of DIR4, the true error seems to be larger than the (official) formal error of $1 \mathrm{~cm}$ at degree 200, given also that the geoid RMS of the comparison of DIR4 to the 675 GPS/levelling observations in Germany is at the same level as TIM4 (Gruber et al, 2013). However, as indicated by the RMS computed with AUS-GGM, the actual DIR4 error is likely to be lower than that of TIM4 at degree 200. In HPF (2013a) independent comparisons to GPS/levelling observations in several countries show RMS values ranging between $1.7 \mathrm{~cm}$ to 3.3 cm, where DIR4 was taken up to d/o 240 and EGM2008 was filled in starting at degree 241 up to $\mathrm{d} / \mathrm{o} 360$.

Compared absolutely in terms of gravity disturbances $\left(T_{r}\right)$, the calculated RMS for DIR4 at degree 200 (1.2 mGal), again, is slightly lower than that of TIM4 (1.3 mGal). In the case of
DIR4 the formal error of $0.35 \mathrm{mGal}$ at degree 200 (own computation) still seems comparatively low to the AUS-GGM RMS. In the case of TIM4, with a formal error of $0.9 \mathrm{mGal}$ at degree 200 (HPF, 2013b), the RMS seems to be realistic, given that the RMS reflects the errors in both data sets.

Compared absolutely in terms of the radial gravity gradients $\left(T_{r r}\right)$, similarly to the other two functionals, the RMS for DIR4 at degree 200 (355 $\mathrm{mE})$ is slightly lower than that of TIM4 (374 mE). Only looking at the $T_{r r}$ formal error estimate for TIM4 at degree 200 (approximately $200 \mathrm{mE}$ ), the RMS values from our analyses seem very high. For TIM4 the formal radial gravity gradient error is exceeded by over $150 \mathrm{mE}$ and it cannot be confirmed by our analyses.

Looking at the lower wavelength part of the spectrum (below d/o 120), the quasi-geoid heights seem to be most sensitive for differences among the models (see bottom row plots in figure 4). Below d/o 120 the TIM3 solution shows the highest RMS. It is followed by TIM4, DIR3 and then by DIR4 with the lowest RMS in that spectral range. Here, obviously, the DIR models which also contain high accuracy GRACE information in the lower degrees agree better with AUS-GGM. Remarkable is the significant improvement of TIM4 w.r.t. TIM3, which are both independent from GRACE, in the bands below degree 150. This will find further investigation and consideration in section 4.2 .

The RMS slope around degree 120 has to be attributed to the comparison model AUS-GGM and not to the GOCE GGFMs, as this is the spectral range where the terrestrial gravity information (with lower accuracy) supersedes GRACE gravity information in AUS-GGM.

In comparison to using EGM2008 for the evaluation of GOCE GGFMs over Australia we found that AUS-GGM shows significantly lower RMS below d/o 150 (meaning a higher agreement with the GOCE models) and similar RMS above degree 150 . To be more precise, EGM2008 shows lower RMS approximately between degree 160 and degree 215 

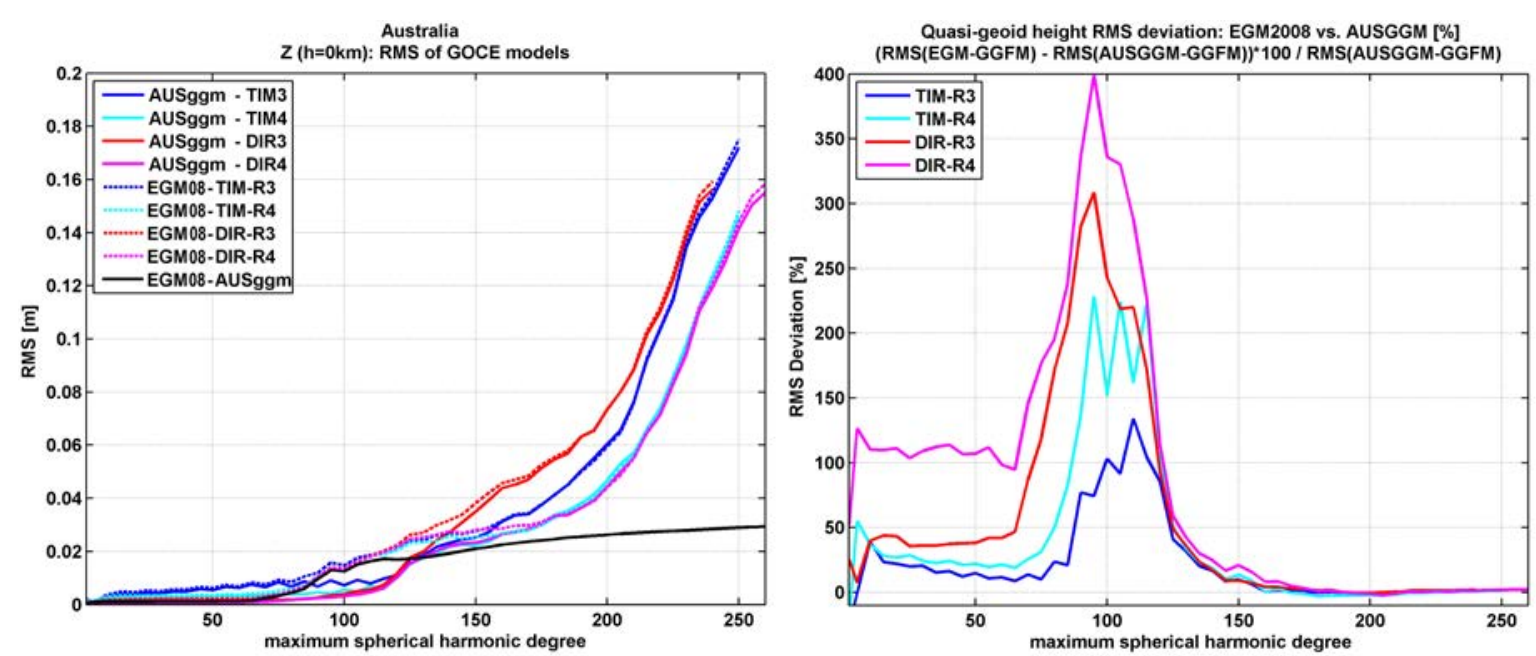

Fig. 5 RMS values over Australia computed from the differences of selected GOCE GGFMs with the newly retrieved AUS-GGM (solid) and EGM2008 (dashed) in terms of quasi-geoid heights in meters [left plot] and the corresponding RMS deviation of EGM2008 w.r.t. AUS-GGM in percent per GOCE GGFM and spherical harmonic degree [right plot]

(depending on the functional; maximum discrepancy of $8.8 \%$ is found for the radial gravity gradient $\left(T_{r r}\right)$ at degree 160$)$. AUS-GGM shows lower RMS values approximately between degree 215 to degree 260. This is shown in figure 5 expressed exemplary in geoid heights (at the ellipsoid). The left plot in figure 5 shows the RMS of GGFMS over Australia w.r.t. AUS-GGM (similar to figure 4a) in solid lines together with the RMS w.r.t. EGM2008 in dashed lines. The right plot only shows the differences of the RMS obtained by EGM2008 w.r.t. AUSGGM per spherical-harmonic degree in percent, where positive values indicate a higher discrepancy of EGM2008 to the respective GOCE GGFM over Australia. The agreement of AUS-GGM with GOCE GGFMs is significantly higher below d/o 120. The better performance of AUS-GGM can partly be explained by using ITG-GRACE2010s instead of ITG-GRACE03 (the latter was used in the EGM2008 creation (Pavlis et al, 2012)). The weaker performance of EGM2008 may also be affiliated with a loss of ITG-GRACE03 information in the model's creation, caused by the weighting applied in the combination with terrestrial data, which was detected over poorly surveyed areas by Hashemi Farahani et al (2013).

At degree 120, we observe a slope in the AUS-GGM produced RMS which comes along with the increasing influence of terrestrial gravity information

\begin{tabular}{c|ccc} 
Difference & $\zeta[\mathrm{cm}]$ & $T_{r}[\mathrm{mGal}]$ & $T_{r r}[\mathrm{E}]$ \\
\hline AUS-GGM - TIM3 & 6.05 & 1.67 & 0.484 \\
AUS-GGM - TIM4 & 4.68 & 1.29 & 0.374 \\
AUS-GGM - DIR3 & 7.34 & 1.99 & 0.569 \\
AUS-GGM - DIR4 & 4.46 & 1.22 & 0.355
\end{tabular}

Table 2 RMS error of GOCE GGFMs w.r.t. AUS-GGM at the spatial scale of $100 \mathrm{~km}$ half wavelength (= degree 200) on the ellipsoid

in the comparison model in this degree range. The fact that quite similar results are achieved with EGM2008 in the degrees beyond 150 we see as a validation of our approach. Keep in mind that the idea of this research to provide methods to produce a GGFM which is regionally completely independent, with up-to date and most accurate terrestrial gravity information. Slightly higher discrepancies to GOCE GGFMs between degree 160 and degree 215 as compared to EGM2008 have to be attributed to errors in the terrestrial gravity data set and the CTM (see section 3.2, 3.3 and 3.4).

\subsection{Evaluation at GOCE altitude}

In this section the RMS values over Australia are computed using the same functionals as in the previous section with the only difference that, now, gravity functionals are calculated at $250 \mathrm{~km}$ altitude above the ellipsoid. With the evaluation at GOCE satellite height we demonstrate the 


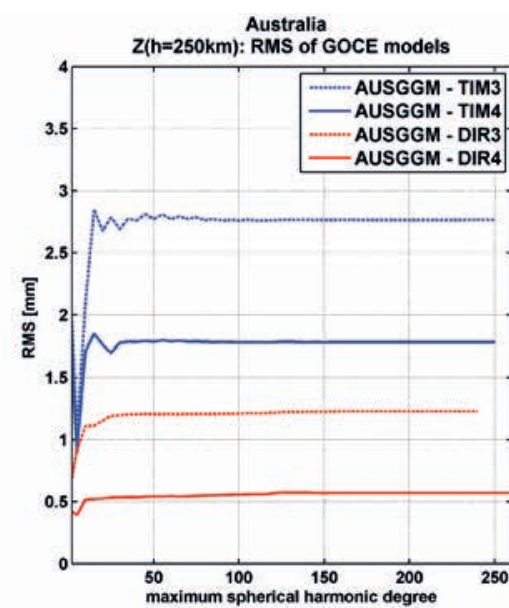

a) quasi-geoid heights

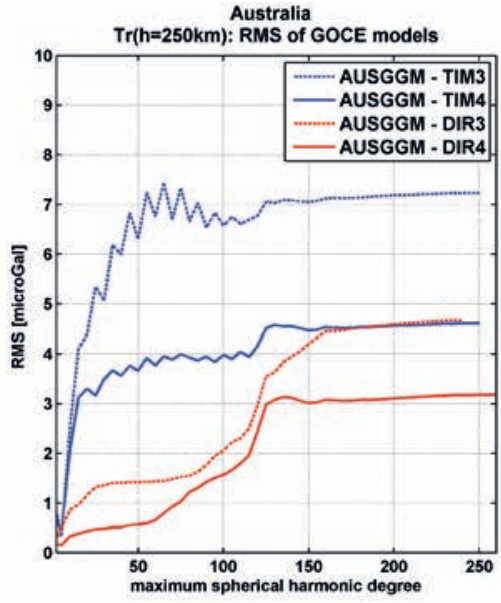

b) gravity disturbances

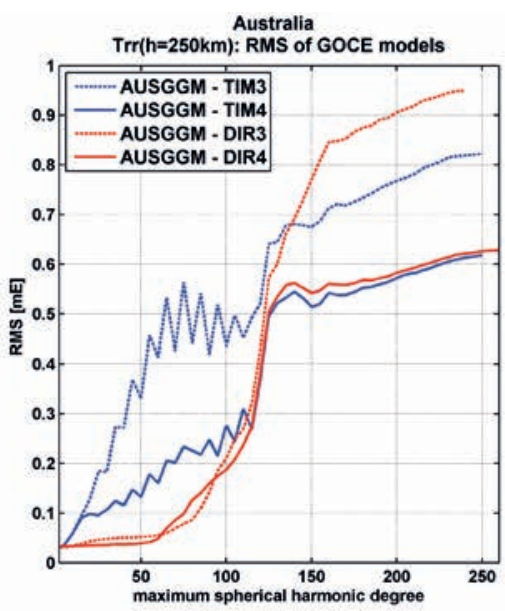

c) radial gravity gradient

Fig. 6 RMS values computed from the differences of selected GOCE GGFMs and the newly retrieved AUS-GGM in terms of (a) quasi-geoid heights $\zeta$ in meters [left], (b) gravity disturbances $T_{r}$ in mGal [middle] and (c) radial gravity gradients $T_{r r}$ [right] $250 \mathrm{~km}$ above the ellipsoid $(h=250 \mathrm{~km})$

attenuation effect and the sensitivity of the functionals at different wavelengths. The results from the evaluation at altitude provide interesting insight into fundamental principles of spectral physical geodesy and allow for some complementary judgment of the models' performance compared to investigations at ground level.

In figure 6 the RMS levels at altitude are generally much lower than those on the ellipsoid (see figure 4), which is due to the attenuation of gravity signals and errors with altitude. At satellite height, the three gravity functionals also show very different features. Starting with the RMS expressed in geoid heights (a), the maximum RMS for each model is already reached at about degree 30, where the slope turns into zero. For gravity disturbances (b) the maximum RMS is reached at degree 160 and for the radial gravity gradient (c) the maximum RMS seems to be reached near degree 230 (as the slope changes near this spectral band). Those findings allow the following categorization concerning the spectral sensitivity of the functionals evaluated at a satellite height of $250 \mathrm{~km}$ : quasi-geoid heights are most sensitive below degree 30; gravity disturbances are most sensitive below degree 160; gravity gradients are most sensitive below degree 230 .

Both fourth generation models show a lower RMS compared to their respective previous release in all three functionals. Looking at the lower wavelength part (below d/o 150), we see again that the DIR models are in better accordance with AUS-GGM because they contain GRACE information in this domain. Further, the interpretation has to be done carefully because the DIR models rely on a different GRACE processing (see section 2) than GRACE data in AUS-GGM (see section 3.3) and the RMS reflects errors in both data sets and/or strategies. However, in all three functionals a clear improvement of the (pure-GOCE) TIM models in the fourth release in the lower wavelength part becomes visible. The three reasons which seem likely to account for this improvement from the third to the fourth TIM release are (1) the change from the energy-integral method (Badura, 2006) to the short-arc method (Mayer-Gürr et al, 2006) in the GOCE SST processing strategy, (2) the improved L1b-processing in the gradiometry (Stummer et al, 2011), and (3) more observations (see table 1). For the other models we can state that DIR4 followed by DIR3 show the lowest discrepancies to AUS-GGM below d/o 150. Interestingly, in the gravity gradients there is a sudden RMS increase at degree 55 for the DIR4 solution (solid red line in figure $6 \mathrm{c}$ ), which is the spherical harmonic degree where the GRACE-GFZ (release 5) supersedes the GRACE-GRGS (release 2) solution in the 


\begin{tabular}{c|ccc} 
Difference & $\begin{array}{c}\zeta \\
{[\mathrm{mm}]}\end{array}$ & $\begin{array}{c}T_{r} \\
{[\text { microGal }]}\end{array}$ & $\begin{array}{c}T_{r r} \\
{[\mathrm{mE}]}\end{array}$ \\
\hline AUS-GGM - TIM3 & 2.77 & 7.18 & 0.768 \\
AUS-GGM - TIM4 & 1.78 & 4.56 & 0.572 \\
AUS-GGM - DIR3 & 1.23 & 4.59 & 0.905 \\
AUS-GGM - DIR4 & 0.57 & 3.11 & 0.582
\end{tabular}

Table 3 RMS error of GOCE GGFMs w.r.t. AUS-GGM at the spatial scale of $100 \mathrm{~km}$ half wavelength (= degree $200)$ at GOCE altitude $(\mathrm{h}=250 \mathrm{~km})$

combination (HPF, 2013a).

Looking at the higher frequency part of the spectrum (beyond degree 150), where AUS-GGM in Australia solely consists of terrestrial data, we see that the RMS values in the quasi-geoid heights and gravity disturbances are at almost constant level and biased mainly due to the differences in the lower frequency part of the spectrum (as stated above the RMS is cumulative, see section 3.5). Those functionals do hardly (gravity disturbances) or not at all (height anomalies) show sensitivity in the spectral domain above d/o 150. The only functional at GOCE altitude that sufficiently allows for discrimination of the GGFM performance at shorter scales are gravity gradients. This sensitivity shown for $T_{r r}$ at GOCE altitude is the very reason for applying gravity gradiometry onboard of GOCE satellite. From the slope of the gravity gradients (beyond degree 150) DIR4 and TIM4 are comparable (same RMS increase per degree) and better (lower RMS increase per degree) than their predecessors. Expressed numerically (calculated from gravity disturbance RMS values retrieved at degree 200 (see table 3)) the relative improvement by the fourth release models at GOCE altitude is $32 \%$ and $36.5 \%$ for the DIR- and TIM-approach, respectively. The relative improvement based on the radial gravity gradient RMS at d/o 200 is $36 \%$ by DIR4 and $25 \%$ by TIM4. Interestingly, in terms of the radial gravity gradient at GOCE altitude, TIM4 for the first time shows a lower RMS than DIR4 in the spectral range between degree 130 and degree 250 .

The estimated formal error in the radial gravity gradient component $T_{r r}$ at GOCE altitude at de- gree 200 is around $0.4 \mathrm{mE}$ and $0.35 \mathrm{mE}$ for TIM3 and TIM4, respectively. Those values are exceeded by the calculated AUS-GGM RMS by $0.36 \mathrm{mE}$ and $0.2 \mathrm{mE}$ (c.f. table 3 ), respectively.

4.3 Discussion on the linkage between the RMS and the Meissl scheme

The Meissl scheme (Rummel and van Gelderen, 1995) establishes the relations between the disturbing potential $T$, its first radial derivative $T_{r}$, and its second radial derivative $T_{r r}$ at ground level $R$ and at altitude $(R+h)$ by means of eigenvalues in the spectral domain. It is, e.g., useful in order to evaluate the design of future gravity missions. Likewise, it can be used to explain the spectral behavior of the RMS of the three functionals on ground level and at satellite height (see figures 4 and 6 ), because it is guide for the spectral characteristics of physical geodesy. The main reason for its applicability to RMS values is, that it does not only apply to the gravity signal, but also to the associated error of derived gravity quantities.

Our evaluations demonstrate different spectral sensitivity in the RMS relying on different functionals. We can categorize the functionals evaluated at a satellite height of $250 \mathrm{~km}$ regarding their sensitivity in the following way: quasi-geoid heights are most sensitive below degree 30; gravity disturbances are most sensitive below degree 160; gravity gradients are most sensitive below degree 230 . This is due to the fact, that the higher part of the spectrum is amplified from the "smoother" to the "rougher" gravity functionals (from left to right in figures 4 and 6). This categorization can not be observed for the RMS values at ground level in the same way. However, quasi-geoid heights are the most sensitive functional in the spectral bands below d/o 50 on the ellipsoid. Further, we find the RMS values at altitude to be smaller, which is due to the increasing attenuation of the signal (and of the error) with increasing distance from the attracting body.

All those features are explained by the Meissl scheme in terms of the eigenvalues (when the 
spherical harmonics are regarded as a set of eigenfunctions). Those eigenvalues we find one-by-one embedded in the SHS algorithms used to expand the spherical harmonic coefficients to the grids which form the basis for the RMS calculation.

\section{Conclusions}

We evaluated the third- and fourth-generation ESA GOCE GGFMs in spherical harmonics and placed focus on a comparison of our evaluation results with the GOCE models' formal errors. The need for an evaluation stems from differences in the processing strategies and in the amount of GOCE data effectively being used in the latest models (DIR3, TIM3 : 12 months; TIM4 : 26.5 months; DIR4 : 27.9 months). We created a spherical harmonic set of coefficients of the disturbing potential which served as an independent reference for the evaluation of GOCE-GGFMs over the landmass of Australia. We made use of the coefficient transformation method, a previously little used but suitable SHA procedure to transform high-frequency terrestrial gravity data into spectral domain. As a result we obtain the comparison model AUSGGM which allows the detection of improvements between the GOCE model releases and, under considerations of its inherent features and errors, can be used to make absolute error estimates. AUSGGM proves to have significantly higher accuracy in the degrees below 120 as compared to EGM2008 and seems to be at least comparable to the accuracy of this model between degree 150 and degree 260. Based on RMS values of three different gravity functionals computed from residual gravity in Australia, we can see a significant improvement of the fourth w.r.t. the third-generation GOCE models. At the ellipsoid, TIM4 and DIR4 are found to show similar RMS values in the high frequency part of the spectrum (beyond degree 120), with the latter performing marginally better between degree 170 to degree 250 which might be linked to one additional month of GOCE gradiometer observations. Relatively, the improvement is about $23 \%$ within the TIM approach and about $39 \%$ within the DIR approach at a spatial scale of $100 \mathrm{~km}$ (at degree 200). At this resolution the models' official formal error expectations in terms of geoid heights is largely confirmed for TIM4 $(3.2 \mathrm{~cm})$, bearing in mind that the comparison data (AUS-GGM) is not free of error. The official DIR4 error estimate of 1 cm (HPF, 2013a) cannot be confirmed, but the error seems to be lower than that of TIM4. In terms of gravity disturbances our RMS of $1.3 \mathrm{mGal}$ for TIM4 (1.2 mGal for DIR4) at degree 200 indicates that also the respective TIM4 error estimate of 0.9 $\mathrm{mGal}$ is quite realistic. Our results can hardly affirm the formal cumulative error of $0.35 \mathrm{mGal}$ (own calculation) of DIR4 at degree 200, even when considering that AUS-GGM is not without errors at those spatial scales.

With the Meissl scheme in hand signal attenuation and spectral sensitivity of the different functionals at different altitude can be explained and the RMS at the 6 different domains of the Meissl scheme help to get a more complete insight into the composition and features of the models. For example, gravity disturbances at satellite altitude clearly demonstrate the improvements of DIR4 and TIM4 in the spectral domain below 150, as compared to the release 3 models. The improvements generally result from a longer period of GOCE observations and changes in the processing strategy of both models. In the fourth DIR release, now, the second CNES/GRGS GRACE solution only finds application in the very low degrees (up to $\mathrm{d} / \mathrm{o}$ 54) and is then superseded by the fifth GFZ GRACE solution. Additionally, the GRACE solutions within DIR4 are based on more data equivalent to 2.5 years of observations. In the fourth TIM release the change from the energy integral approach to the short-arc integral method in the SST processing explains a large part of the improvement in the long wavelength part of the spectrum. Further, both, TIM4 and DIR4 benefit from a new L1b-processing procedure for GOCE gradients.

From our evaluations we conclude that with the fourth-generation GOCE models a better knowledge of the Earth's gravity field in poorly surveyed 
areas (e.g. parts of South America, Africa, and Asia) at spatial scales of $80 \mathrm{~km}$ up to $120 \mathrm{~km}$ is to be expected.

Acknowledgements This study was supported by the Australian Research Council (Grant DD12044/100) and through funding from Curtin University's Office of Research and Development. We would like to thank Thomas Gruber for suggesting evaluations in accordance with the Meissl scheme. We thank Will Featherstone for providing the Australian gravity grids. We highly acknowledge the efforts of ESA and the HPF team for providing GOCE products in a very convenient and timely manner.

\section{References}

Abdalla A, Fashir H, Ali A, Fairhead D (2012) Validation of recent GOCE/GRACE geopotential models over Khartoum state - Sudan. Journal of Geodetic Science 2:88-97, DOI 10.2478/v10156011-0035-6

Andersen O, Knudsen P, Berry P (2009) The DNSC08GRA Global Marine Gravity field from Double Retracked Satellite Altimetry. Journal of Geodesy 84:191-199, DOI 10.1007/s00190-0090355-9

Badura T (2006) Gravity field analysis from satellite orbit information applying the energy integral approach. PhD thesis, Graz University of Technology

Bouman J, Fuchs M (2012) GOCE gravity gradients versus global gravity field models. Geophysical Journal International 189:846-850, DOI doi: 10.1111/j.1365-246X.2012.05428.x

Bruinsma S, Lemoine J, Biancale R, Valès N (2010a) CNES/GRGS 10-day gravity field models (release 2) and their evaluation . Advances in Space Research 45(4):587 - 601, DOI 10.1016/j.asr.2009.10.012

Bruinsma S, Marty J, Balmino G, Biancale R, Förste C, Abrikosov O, Neumayer H (2010b) GOCE gravity field recovery by means of the direct numerical method. In: Lacoste-Francis H (ed) Proceedings of the ESA Living Planet Symposium, 28.June-2.July, Bergen, ESA Publication SP-686
Chen J, Wilson C (2010) Assessment of Degree2 Zonal Gravitational Changes from GRACE, Earth Rotation, Climate Models, and Satellite Laser Ranging. In: Mertikas S (ed) Gravity, Geoid and Earth Observation, Springer Berlin / Heidelberg, vol 135, pp 669-676, DOI 10.1007/978-3-642-10634-7_88

Claessens S (2006) Solutions to Ellipsoidal Boundary Value Problems for Gravity Field Modelling. $\mathrm{PhD}$ thesis, Curtin University of Technology

Claessens S, Featherstone W, Anjasmar I, Filmer M (2009) Is Australian data really validating EGM2008, or is EGM2008 just in/validating Australian data? In: Newton's Bulletin Issue no 4, International Association of Geodesy and International Gravity Field Service, pp 207-251

Dahle C, Flechtner F, Gruber C, König D, König R, Michalak G, Neumayer K (2013) GFZ GRACE Level-2 Processing Standards Document for Level-2 Product Release 0005: revised edition., GeoForschungsZentrum Potsdam

Driscoll J, Healy D (1994) Computing Fourier Transforms and Convolutions on the 2-Sphere. Advances in Applied Mathematics 15(2):202250, DOI 10.1006/aama.1994.1008

ESA (1999) Gravity Field and Steady-State Ocean Circulation Mission. Report for the mission selection of the four candidate earth explorer missions (ESA SP-1233(1)), European Space Agency

Featherstone W, Kirby J (2000) The reduction of aliasing in gravity anomalies and geoid heights using digital terrain data. Geophysical Journal International 141:204-212

Featherstone W, Kirby J, Hirt C, Filmer M, Claessens S, Brown N, Hu G, Johnston G (2010) The AUSGeoid model of the Australian Height Datum. Journal of Geodesy 85(3):133-150

Forsberg R, Kenyon S (2004) Gravity and Geoid in the Arctic Region - The Northern Polar Gap now Filled. In: Proceedings of the 2nd GOCE User Workshop, March 2004, Esrin

Gerlach C, Šprlák M, Bentel K, Pettersen B (2013) Observation, Validation, Modeling - Historical Lines and Recent Results in Norwegian Gravity 
Field Research. Kart og Plan 73(2):128 - 150

Gruber T, Visser P, Ackermann C, Hosse M (2011) Validation of GOCE gravity field models by means of orbit residuals and geoid comparisons. Journal of Geodesy 85(11):845-860, DOI 10.1007/s00190-011-0486-7

Gruber T, Rummel R, HPF-Team (2013) The 4th Realease of GOCE Gravity Field Models - Overview and Performance. In: Presentation at EGU General Assembly 2013, http://www.iapg.bv.tum.de/mediadb/5533197/ 5533198/20130411_EGU_Gruber_GOCE.pdf

Guimarães G, Matos A, Blitzkow D (2012) An evaluation of recent GOCE geopotential models in Brazil. Journal of Geodetic Science 2:144-155, DOI 10.2478/v10156-011-0033-8

Hashemi Farahani H, Ditmar P, Klees R, Teixeria da Encarnacao J, Liu X, Zhao Q, Guo J (2013) Validation of static gravity field models using GRACE K-band ranging and GOCE gradiometry data. Geophysical Journal International 194:751-771, DOI 10.1093/gji/ggt149

Hirt C, Gruber T, Featherstone W (2011) Evaluation of the first GOCE static gravity field models using terrestrial gravity, vertical deflections and EGM2008 quasigeoid heights. Journal of Geodesy 85:723-740

Hirt C, Kuhn M, Featherstone W, Göttl F (2012) Topographic/isostatic evaluation of newgeneration GOCE gravity field models. Journal of Geophysical Research - Solid Earth 117(B05407), DOI 10.1029/2011JB008878.

Holmes S, Pavlis N (2008) EGM Harmonic Synthesis Software. , National Geospatial-Intelligence Agency, http://earth-info.nga.mil /GandG /wgs84 /gravitymod / new_egm /new_egm.html HPF (2013a) Datasheet GO_CONS_GCF_2 _DIR_R4. , GFZ, GRGS, published at ICGEM

HPF (2013b) Datasheet GO_CONS_GFC_2 _TIM_R4. , Graz University of Technology, University of Bonn,TU München, published at ICGEM

Ihde J, Wilmes H, Müller J, Denker H, Voigt C, Hosse M (2010) Validation of satellite gravity field models by regional terrestrial data sets.
In: Flechtner F, Gruber T, Güntner A, Mandea M, Rothacher M, Schöne T, Wickert J (eds) System Earth via Geodetic-Geophysical Space Techniques, Springer, pp 277-296, DOI 10.1007/978-3-642-10228-8_22

Janák J, Pitoňák M (2011) Comparison and testing of GOCE global gravity models in Central Europe. Journal of Geodetic Science 1:333-347

Jekeli C (1988) The exact transformation between ellipsoidal and spherical harmonic expansions. manuscripta geodaetica 13(2):106-113

Kaula W (1966) Theory of Satellite Geodesy. Blaisdel, Waltham

Lavallée D, Moore P, Clarke P, Petrie E, van Dam T, King M (2010) J2: An evaluation of new estimates from GPS, GRACE, and load models compared to SLR. Geophysical Research Letters 37, DOI 10.1029/2010GL045229

Mayer-Gürr T, Eicker A, Ilk KH (2006) Gravity Field Recovery from GRACE-SST Data of Short Arcs. In: Flury J, Rummel R, Reigber C, Rothacher M, Boedecker G, Schreiber U (eds) Observation of the Earth System from Space, Springer Berlin / Heidelberg, pp 131-148

Mayer-Gürr T, Kurtenbach E, Eicker A (2010) ITG-Grace2010 Gravity Field Model., www.igg. uni-bonn.de/apmg/index.php?id=itg-grace2010

Metzler B, Pail R (2005) GOCE data processing: the spherical cap regularization approach. Studia Geophysica et Geodaetica 49(4):441-462, DOI 10.1007/s11200-005-0021-5

Migliaccio F, Reguzzoni M, Sanso F, Tscherning C, Veicherts M (2010) GOCE data analysis: the space-wise method approach and the first spacewise gravity field model. In: Proceedings of the ESA Living Planet Symposium, 28.June-2.July, Bergen, ESA Publication SP-686

Moritz H (2000) Geodetic Reference System 1980. Journal of Geodesy 74(1):128-162

Pail R, Goiginger H, Mayrhofer R, Schuh WD, Brockmann JM, et al (2010) GOCE gravity field model derived from orbit and gradiometry data applying the Time-Wise Method. In: LacosteFrancis $\mathrm{H}$ (ed) Proceedings of the ESA Living Planet Symposium, 28.June-2.July, Bergen, 
ESA Publication SP-686

Pail R, Bruinsma S, Migliaccio F, Förste C, Goiginger H, Schuh WD, Höck E, Reguzzoni M, Brockmann JM, Abrikosov O, Veicherts M, Fecher T, Mayrhofer R, Krasbutter I, Sanso F, Tscherning CC (2011a) First GOCE gravity field models derived by three different approaches. Journal of Geodesy 85(11):819-843, DOI 10.1007/s00190011-0467-x, special issue: "GOCE - The Gravity and Steady-state Ocean Circulation Explorer"

Pail R, Goiginger H, Schuh W, Höck E, Brockmann J, Fecher T, Mayer-Gürr T, Kusche J, Jäggi A, Rieser D, Gruber T (2011b) Combination of GOCE data with complementary gravity field information (GOCO). In: Proceedings of 4th International GOCE User Workshop, Munich, 31st March 2011, ESA SP-696, Noordwijk

Pail R, Fecher T, Murböck M, Rexer M, Stetter M, Gruber T, Stummer C (2012) Impact of GOCE L1b data reprocessing on GOCEonly and combined gravity field models. In: Studia Geophysica et Geodaetica, Springer, DOI $10.1007 / \mathrm{s} 11200-012-1149-8$

Pavlis N, Holmes S, Kenyon S, Factor J (2012) The developement and evaluation of the Earth Gravitational Model 2008 (EGM2008). Journal of Geophysical Research 117, DOI 10.1029/2011JB008916

Rummel R, van Gelderen M (1995) Meissl scheme - spectral characteristics of physical geodesy. Manusrcipta Geodaetica 20:379-385

Sneeuw N, van Gelderen M (1997) The polar gap. In: Geodetic Boundary Value Problems in view of the One Centimeter Geoid. Lecture Notes in Earth Science 65:559-568

Šprlák M, Gerlach C, Omang O, Pettersen B (2011) Comparison of GOCE derived satellite global gravity models with EGM2008, the OCTAS geoid and terrestrial gravity data: case study for Norway. In: Proceedings of the 4th International GOCE User Workshop, Munich, 31st March 2011, ESA SP-696, Noordwijk

Šprlák M, Gerlach C, Pettersen B (2012) Validation of GOCE global gravity field models using terrestrial gravity data in Norway. Journal of Geodetic Science 2(2):134-143

Stummer C, Pail R, Fecher T (2011) Alternative method for angular rate determination within the GOCE gradiometer processing. Journal of Geodesy Vol. 85 (9):585-596

Stummer C, Siemes C, Pail R, Frommknecht B, Floberghagen R (2012) Upgrade of the GOCE Level 1b gradiometer processor. Advances in Space Research 49 (4):739-752

Szücz E (2012) Validation of GOCE time-wise gravity field models using GPS-levelling, gravity, vertical deflections and gravity gradients in Hungary. Civil Engineering 56(1):3 - 11

Tapley B, Reigber C (2001) The grace mission: status and future plans. In: AGU Fall Meeting Abstracts, vol 1, p 02

Tapley B, Schutz B, Eanes R, Ries J, Watkins M (1993) LAGEOS laser ranging contributions to geodynamics, geodesy, and orbital dynamics. GEODYNAMICS SERIES 24:147-173

Torge W (2001) Geodesy, 3rd edn. Walter de Gruyter

Tscherning C, Arabelos D (2011) Gravity anomaly and gradient recovery from GOCE gradient data using LSC and comparisons with known ground data. In: Proceedings 4th International GOCE user workshop, Munich, 31st March 2011, ESA SP-696, Noordwijk

Voigt C, Denker H (2011) Validation of goce gravity field models by astrogeodetic vertical deflections in germany. In: Proceedings of the 4 th International GOCE User Workshop, SP-696, ESA/ESTEC, The Nederlands, vol 4

Voigt C, Rülke A, Denker H, Ihde J, Liebsch G (2010) Validation of goce products by terrestrial data sets in germany. In: Observation of the System Earth from Space, Geotechnolgien, Potsdam/Germany, vol 17

Wang Y (1989) Downward Continuation of the Free-Air Gravity Anomalies to the Ellipsoid Using the Gradient Solution, Poisson's Integral and Terrain-Correction - Numerical Comparison and Computations. 4, Department of Geodetic Science and Surveying, Ohio State University 
Zwally H, Schutz B, Abdalati W, Abshire J, Bentley C, Brenner A, Bufton J, et al (2002) ICESat's laser measurement of polar ice, atmosphere, ocean, and land. Journal of Geodynamics 34:404-445 